\title{
Quantifying muscle tone in spinal cord injury patients using isokinetic dynamometric techniques
}

\author{
K Perell ${ }^{1,2}$, A Scremin ${ }^{1,3}$, O Scremin ${ }^{1,4}$ and C Kunkel ${ }^{5}$ \\ ${ }^{1}$ Physical Medicine and Rehabilitation Service, West Los Angeles VAMC, Los Angeles, CA 90073, USA; \\ ${ }^{2}$ Department of Physiological Science, UCLA, Los Angeles, CA 90024, USA; ${ }^{3}$ Department of Medicine-PM\&R \\ Division, UCLA School of Medicine, Los Angeles, CA 90024, USA; ${ }^{4}$ Geriatric, Research and Education Clinical \\ Center (GRECC), West Los Angeles VAMC, Los Angeles, CA 90073 and Department of Physiology, UCLA School \\ of Medicine, Los Angeles, CA 90024, USA; ${ }^{5}$ Physical Medicine and Rehabilitation Service, Albuquerque VAMC, \\ Albuquerque, NM 87180 and Department of Orthopedics, University of New Mexico, Albuquerque, NM 87180, USA
}

The torque generated during a passive movement of the knee joint was used to quantify muscle tone in normal able-bodied subjects and spastic and flaccid spinal cord injury (SCI) subjects using a computerized isokinetic dynamometer. Maximum peak $\left(\mathrm{T}_{\max }\right)$ and the sum of four consecutive peaks $\left(\mathrm{T}_{\text {sum }}\right)$ were calculated for each velocity $\left(30,60,120^{\circ} / \mathrm{s}\right)$ and for each phase (flexing or extending) separately and compared statistically using a one-way ANOVA. Statistical significance between groups was found in $T_{\max }$ FLEXION (FLX) at 60 and $120^{\circ} / \mathrm{s}$. Scheffe's tests revealed that the spastic group was significantly less than both the flaccid and normal groups, although the flaccid and normal groups were not significantly different from each other. The slopes of the linear regression curve of the torque-velocity data were found and compared statistically using a $t$-test for parallelism. In all parameters, the data increased in a linear fashion with increasing velocity of knee motion. The slope of the regression curve for the spastic group was significantly lower than that of the normal group for $T_{\max }$ and was significantly lower than that of the flaccid group for $T_{\text {sum }}$ while the slopes for the flaccid and normal groups were not significantly different. The ability of the entire set of variables to classify subjects into three groups (normal, spastic, and flaccid) was tested using discriminant analysis. By taking into account 7 of the 12 original variables, this multivariate technique correctly classified $100 \%$ of the spastic, $90 \%$ of the normal, but only $67 \%$ of the flaccid subjects. Separation of observations was between spastic and normal subjects was good, except for only one case. This feature could be useful when dealing with assessment of individual responses to therapeutic interventions aimed at modification of spasticity.

Keywords: muscle tone; spinal cord injury; isokinetic dynamometer

\section{Introduction}

In the treatment of spasticity, interventions including drug therapy are often effective if they can reduce the level of abnormal muscle tone. Therefore, methods of accurately measuring muscle tone are important in defining the magnitude of the abnormality, and in quantifying the effectiveness of therapeutic interventions aimed at restoring normal tone. The ability to quantify the presence and severity of muscle tone is essential to the understanding and treatment of this disorder. Types of testing to quantify muscle tone are divided into three categories: clinical measures such as passive manual stretch or the Ashworth scale ${ }^{2}$ which while simple to perform, lack precision and objectivity; neurophysiological measures such as tendon jerk, $\mathrm{H}$ reflex, and tonic vibration reflex which have weak correlation between each other and with the clinical

Correspondence: K Perell, Physical Medicine \& Rehabilitation Service (117), West Los Angeles VAMC, 11301 Wilshire Blvd., Los Angeles, CA 90073, USA status of muscle tone; $;^{3,4}$ and biomechanical measures such as the gravitational or pendulum methods which are simple but does not control the force or movement parameters, ${ }^{5,6,7,8}$ the controlled torque method which imposes a torque on the limb and measures the movement displacement and velocity $1,9,10$ and the controlled displacement methods which impose a movement and measure the force developed. $4,8,11,12$ The advantage of the controlled displacement methods is that velocity and range of motion can be standardized and controlled. The ability to vary velocities allows for the evaluation of the ratedependent characteristics of muscle tone.

We have recently reported on a technique using the controlled displacement method to quantify muscle tone by determining the summation of four consecutive resistive torque amplitudes during flexion and extension of the knee at specified speeds and range of motion using an isokinetic dynamometer (Kin-Com 
$500 \mathrm{H}$ ). Specific details regarding the technique are described in that report. ${ }^{4}$ Our results indicated that the summation of four consecutive resistive torque amplitudes and the determination of the slopes of the torque-velocity curves are specific and reproducible methods of differentiating individuals with spasticity from those with normal muscle tone.

Since our initial study ${ }^{4}$ did not include flaccid subjects, we were unable to determine the usefulness of this technique in differentiating between subjects with spasticity and flaccidity, or flaccidity and normal muscle tone. Furthermore, the initial population of spastic subjects was heterogeneous including individuals with spinal cord injury, stroke and anterior horn cell disease. The present study was designed to include a population consisting solely of spinal cord injured subjects with clinically apparent spasticity or flaccidity, and a group of subjects with normal muscle tone.

\section{Methods}

The study group consisted of 11 spastic SCI subjects, 6 flaccid SCI subjects, and 10 able-bodied subjects with normal tone (normal group). All subjects were medically and neurologically stable. The spastic subjects had muscle tone varying in degree from mild to severe based on the physical examination (Ashworth scale $=2-4){ }^{2}$ Lack of muscle tone in the flaccid subjects was confirmed by physical examination (Ashworth scale $=0) .{ }^{2}$ Groups were not significantly different for age or weight (Table 1). Years post injury between the spastic (Mean \pm SD: $9.00 \pm 5.80$ ) and flaccid (Mean \pm SD: $17.67 \pm 8.63$ ) groups were significantly different $(P=0.03)$. Level of injury ranged from C5-L1 for the spastic group (3 patients with tetraplegia) and $\mathrm{T} 11-\mathrm{L} 1$ for the flaccid group.

A commercially available computerized isokinetic dynamometer (Kin-Com model 500H, Chattacx Co., Chattanooga, TN) was used. The evaluation mode menu with high acceleration and deceleration was used. The choice of high acceleration (or deceleration) controlled a quick rate at which the machine accelerated (or decelerated) up to the preselected velocity during the turning points or transitions of the lever from flexing to extending or vice versa. The force applied to the lever during the passive movement of the knee joint through a $50^{\circ}$ range of motion $\left(25^{\circ}(0.44 \mathrm{rad})\right.$ flexion to $75^{\circ}(1.32 \mathrm{rad})$ flexion $)$ at preset angular velocities of 30,60 , and $120 \mathrm{deg} / \mathrm{s}$ $(0.53,1.05$, and $2.11 \mathrm{rad} / \mathrm{s})$ was recorded for varied

Table 1 Group means \pm standard deviations for age and weight

\begin{tabular}{lccc}
\hline Group & Number of subjects & Age (years) & Weight $(\mathrm{kg})$ \\
\hline Spastic & 11 & $36 \pm 6$ & $75.9 \pm 12.1$ \\
Flaccid & 6 & $45 \pm 8$ & $83.7 \pm 17.7$ \\
Normal & 10 & $33 \pm 12$ & $75.5 \pm 8.3$ \\
\hline
\end{tabular}

lengths of time ranging from 20 to $40 \mathrm{~s}$ depending on the velocity setting which corresponded to $10-12$ repetitions.

Each subject was seated on a chair with thighs and hips restrained by straps and the legs hanging over the edge of the seat (Figure 1). The hip was maintained at $90^{\circ}$ flexion. The rotation axis of the dynamometer was set to correspond with the rotational axis of the knee joint. Attachment of the lower extremity to the lever of the dynamometer was made $4 \mathrm{~cm}$ above the lateral malleolus. The posterior portion of the shank rested on the front of the lever and was anchored tightly to the lever with velcro straps. To account for the contribution of the limb's weight, a gravity correction was performed as part of the isokinetic dynamometer software.

Analysis was done using the 5 middle repetitions of the series of $10-12$ repetitions at each speed. The first portion of the repetition (from start angle (Figure 2, point A) to stop angle (Figure 2, point B)), was defined as the flexing phase. The second portion of the repetition (from stop angle (Figure 2, point $\mathrm{B}$ )) to start angle (Figure 2, point $\mathrm{C}$ )) was defined as the extending phase. Positive forces represent pushing against the lever in either phase. Negative forces represent pulling away from the lever in either phase.

The sum of four consecutive absolute 'peak' forces (denoted as F1, F2, F3, F4 or E1, E2, E3, \& E4 in Figure 2) ( $\mathrm{T}_{\text {sum }}$ FLX $=|\mathrm{FI}|+|\mathrm{F} 2|+|\mathrm{F} 3|+|\mathrm{F} 4|$ and

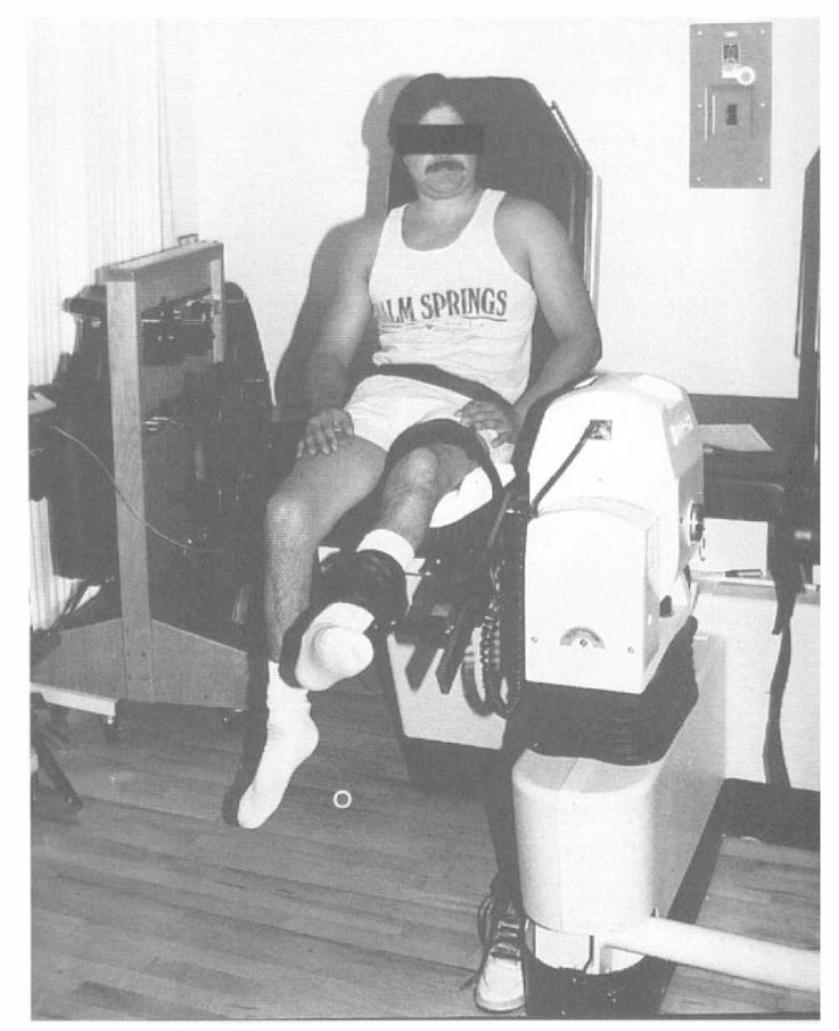

Figure 1 Body position of subject on isokinetic dynamometer 


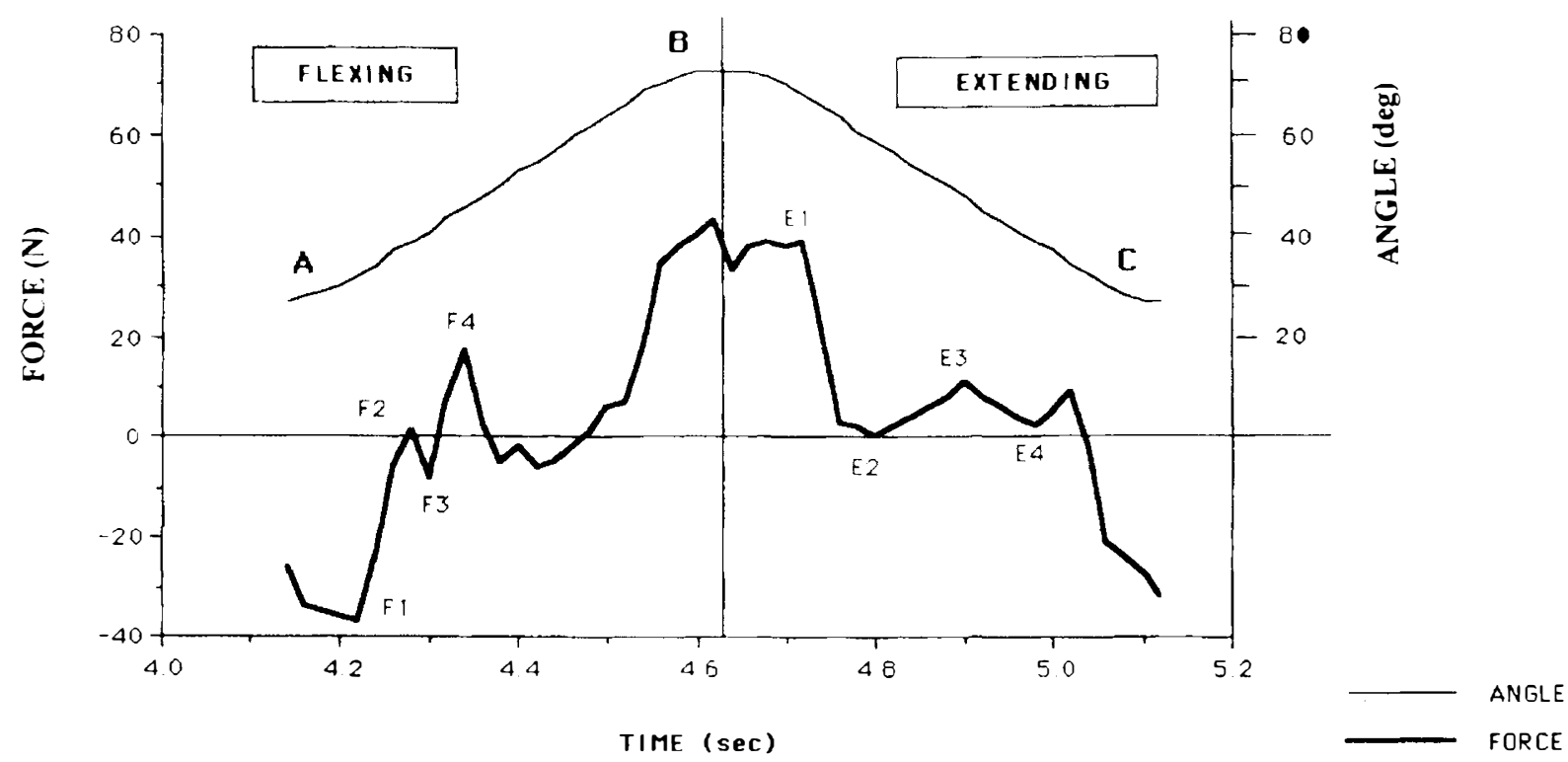

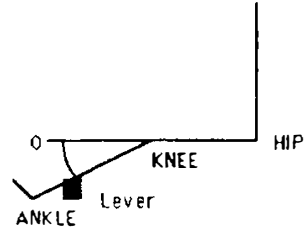

A 25 degrees or flexion

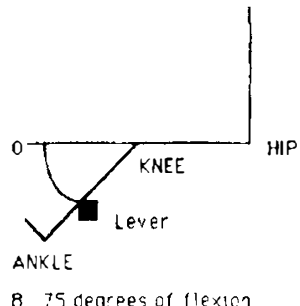

875 degrees of flexion

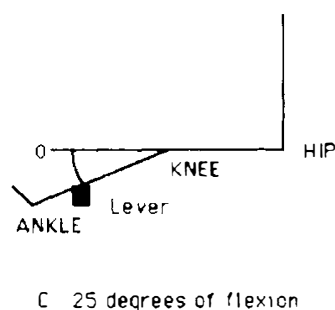

Figure 2 Raw angle and force time data for one repetition and notation of peak force values (top graph). Schematic drawing of limb and force lever through the range of motion at stop and start angles (bottom graph). This is exemplar data from one spastic subject

$\left.\mathrm{T}_{\text {sum }} \mathrm{EXT}=|\mathrm{E} 1|+|\mathrm{E} 2|+|\mathrm{E} 3|+|\mathrm{E} 4|\right)$ within a repetition was calculated for the 5 middle repetitions for each phase (flexing or extending) separately. The consecutive absolute 'peak' forces refer to the maximum peak force and the following three changes in the direction of force. These may result in minimums as well as maximums. The average torque over the 5 middle repetitions for the maximum peak $\left(\mathrm{T}_{\max }\right)$ and the sum of four consecutive peaks $\left(\mathrm{T}_{\text {sum }}\right)$ was calculated for each velocity $\left(30,60,120^{\circ} / \mathrm{s}\right)$ and for each phase (flexing or extending) separately. Statistical significance for $T_{\max }$ and $T_{\text {sum }}$ was determined by a one-way ANOVA. Scheffe's tests were used to distinguish differences between groups. The significance level used throughout the study was $P<0.05$. The slopes of the linear regression curve of the torque-velocity data were found and compared statistically between the groups using a $t$-test for parallelism.

A discriminant analysis ${ }^{14}$ was performed for the dependent classification variable muscle tone, with 3 discrete values (Spastic, Flaccid and Normal) and 12 continuous independent variables that included $\mathrm{T}_{\max }$ and $\mathrm{T}_{\text {sum }}$ for extension and flexion at 30, 60, and $120 \mathrm{deg} / \mathrm{s}$.
The linear discriminant function technique was used for classification. Variables with an F-value $<1$ were removed from the discriminant equation. The three linear discriminant scores of every observation (obtained when the independent variable values were used with the corresponding linear discriminant equations) were transformed to the estimated probabilities of that observation belonging to each specific group.

All statistical analyses were performed with the NCSS software package (NCSS, Kaysville, Utah).

\section{Results}

Means and standard deviations of $T_{\max }$ and $T_{\text {sum }}$ are graphically presented in Figure $3 \mathrm{~A}-\mathrm{D}$. While there was a trend toward lower values in the spastic group compared to the other two groups, statistical significance was found only in $\mathrm{T}_{\max } \mathrm{FLX}$ at $60^{\circ} / \mathrm{s}(P=0.0009)$ and $120^{\circ} / \mathrm{s}$ $(P=0.0017)$. At $60^{\circ} / \mathrm{s}$, the mean $\pm \mathrm{SD}$ for the spastic group $\mathrm{T}_{\max } \mathrm{FLX}=8.00 \pm 4.35 \mathrm{~N}^{*} \mathrm{~m}$, the flaccid group $\mathrm{T}_{\max } \mathrm{FLX}=16.68 \pm 6.84 \mathrm{~N}^{*} \mathrm{~m}$, and the normal group $\mathrm{T}_{\max } \mathrm{FLX}=16.63 \pm 4.10 \mathrm{~N}^{*} \mathrm{~m}$. At $120^{\circ} / \mathrm{s}$, the mean $\pm \mathrm{SD}$ for the spastic group $T_{\max } F L X=19.00 \pm 7.11 \mathrm{~N}^{*} \mathrm{~m}$, the flaccid group $\mathrm{T}_{\max } \mathrm{FLX}=34.31 \pm 9.74 \mathrm{~N}^{*} \mathrm{~m}$, and the 


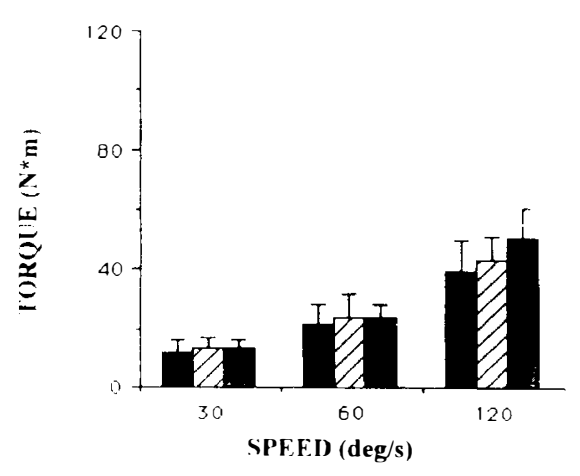

C

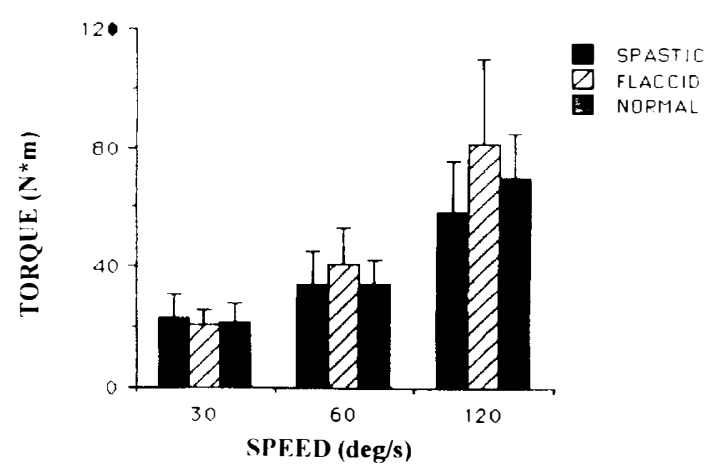

B

Tmax Flexion

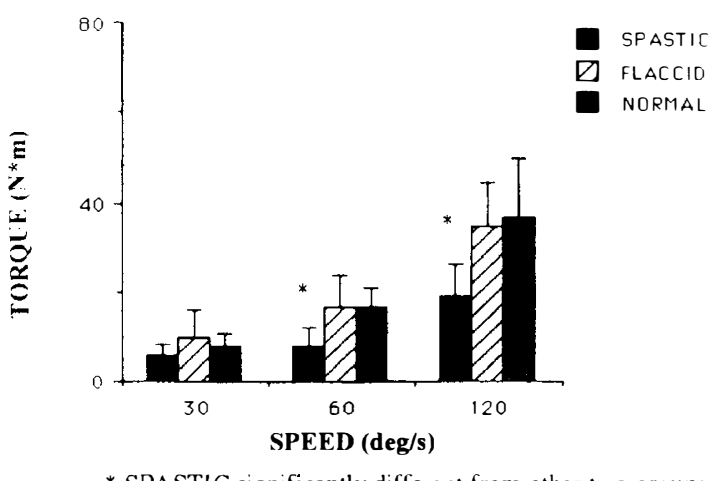

* SPASTIC significantly different from other two groups

D

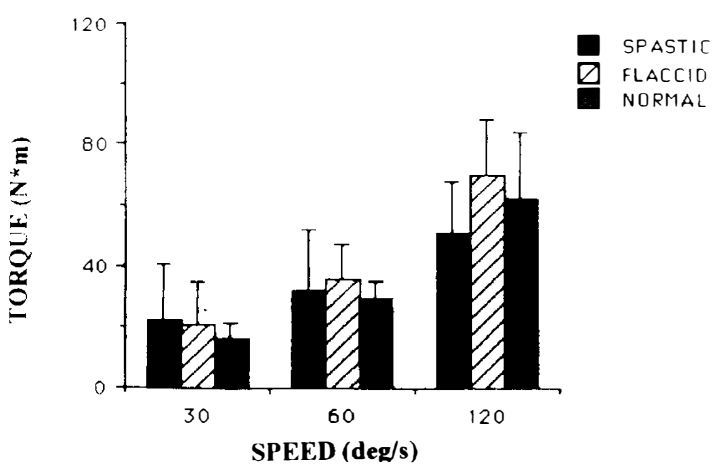

Figure 3 Graphical representation of the mean \pm standard deviation for (A) $T_{\max }$ EXT, (B) $T_{\max }$ FLX, (C) $T_{\text {sum }}$ EXT, and (D) $\mathrm{T}_{\text {sum }}$ FLX for the spastic, flaccid, and normal groups

normal group $\mathrm{T}_{\max } \mathrm{FLX}=36.49 \pm 13.13 \mathrm{~N} * \mathrm{~m}$. Scheffe's tests revealed that the spastic group was significantly less than both the flaccid and normal groups, although the flaccid and normal groups were not significantly different from each other.

Regression analysis demonstrated that $\mathrm{T}_{\max }$ and $\mathrm{T}_{\text {sum }}$ in both directions increased linearly $(P<0.05)$ with velocity of knee motion (Figure 4A-D). The slope of the regression curve was smallest in the spastic group and largest in the normal group for $T_{\max }$ in both directions. The slope of the regression curve was smallest in the spastic group and largest in the flaccid group for $\mathrm{T}_{\text {sum }}$ in both directions. The slope of the regression curve for the spastic group was significantly lower than that of the normal group for $T_{\max }$ and was significantly lower than that of the flaccid group for $\mathrm{T}_{\text {sum. }}$. Only for $\mathrm{T}_{\max }$ FLX was the slope of the regression curve for the spastic group significantly lower than that of both groups. The curves for the flaccid and normal groups were not significantly different for any parameter.

Discriminant analysis showed that 7 of the 12 variables studied contributed significantly to group classification. These variables are listed in Table 2, which also shows the linear discriminant functions for each group, and the F-values and probabilities. Good predictability was implied by an overall Wilks lambda of 0.1659 . The classification matrix (Figure 5, right upper quadrant) showed that $100 \%$ of spastic subjects and $90 \%$ of normal subjects were correctly classified by this procedure. Classification of subjects in the flaccid group was less efficient (only 4 out of 6), a fact probably related to the smaller number of subjects in that group.

The predicted probabilities of observations belonging to each specific group are depicted graphically in Figure 5 for the three possible contrasts (Spastic vs Normal, Flaccid vs Normal, and Spastic vs. Flaccid). Excellent clustering of normal observations close to a predicted probability of 1 for normal and 0 for spastic was obtained when these two parameters were plotted against each other (Figure 5, left upper quadrant). Similar, although less tight, distribution was observed for the spastic group in that plot. Separation of groups was also good between flaccid and normal (left lower quadrant), while some overlap existed between spastic and flaccid (right lower quadrant).

\section{Discussion}

Unlike other studies which compared spinal cord injured (SCI) to normal able-bodied subjects, this study compared SCI patients who were determined to be spastic (based on the Ashworth test) to those SCI 

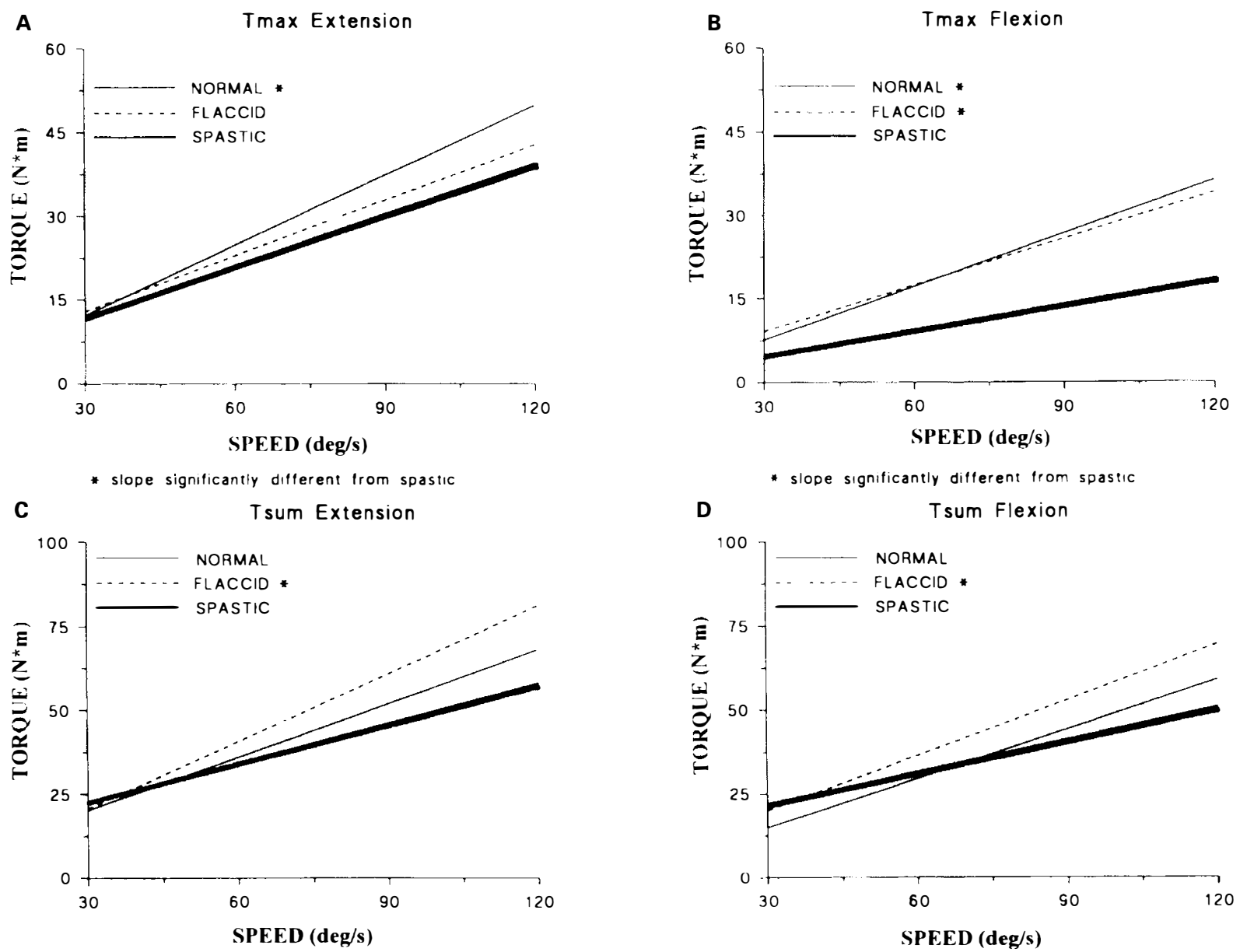

Figure 4 Regression line for torque versus velocity curves of (A) $T_{\max }$ EXT, (B) $T_{\max }$ FLX, (C) $T_{\text {sum }}$ EXT, and (D) $T_{\text {sum }}$ FLX for the spastic, flaccid, and normal groups

patients who were determined to be flaccid, as well as to normal able-bodied subjects. Furthermore, this investigation studied a homogenous group of complete SCI patients in the spastic group. Other studies have used a heterogeneous population including individuals with SCI, stroke, and anterior horn cell disease ${ }^{4}$ or incomplete and complete SCI patients. ${ }^{12}$

The most significant finding in this study was that $\mathrm{T}_{\max }$ FLX could be used to distinguish a homogenous population of complete, spastic SCI subjects from flaccid SCI subjects, and from normal able-bodied subjects. $\mathrm{T}_{\max }$ FLX of the spastic group was significantly lower at 60 and $120^{\circ} / \mathrm{s}$ than that of the other two groups. Consequently, the slope of the regression line for the spastic group was significantly smaller than that of the other two groups. Since the lower speed did not demonstrate differences between groups and the slope of the regression line across the three speeds did not show any additional results from that obtained using $\mathrm{T}_{\max }$, using only $\mathrm{T}_{\max }$ at $120^{\circ} / \mathrm{s}$ as a clinical test may be sufficient especially considering the extensive data analysis necessary to obtain the other parameters.
The decreased $T_{\max }$ FLX for the spastic group was a surprising finding. It was expected, as has been shown by others, ${ }^{4,12}$ that the spastic group would have higher resistance to movement and consequently larger resistive forces than the normal group because of increased muscle tone in spastic SCI subjects. Measures of muscle stiffness, however, support our findings that values are lower in spastic subjects than in normals. ${ }^{9}$ Stiffness is defined as torque divided by angular displacement. While Douglas et al. ${ }^{9}$ used the controlled torque method, holding torque constant and measuring angular displacement, we used the controlled displacement method, holding angular displacement constant and measuring torque. In either case, muscle stiffness was lowest in the spastic group.

While Douglas et al. ${ }^{9}$ attribute the reduction in muscle stiffness to muscle atrophy, we believe, instead, that a decreased $T_{\max }$ FLX which was significantly different from both the normal and flaccid groups may represent not the increased muscle tone experienced by spastic SCI subjects, but rather a kinetic measure analogous to the relaxation index of the pendulum 
Table 2 Linear discriminant functions and $F$ values and probabilities for the variables included in the discriminant analysis

\begin{tabular}{lrrrrrr}
\hline Variable & Spastic & Flaccid & Normal & F-value & F & probability \\
\cline { 1 - 4 } $\mathrm{T}_{\text {max }}-\mathrm{E} 120$ & 0.41 & 0.37 & 1.07 & 13.30 & 0.0002 \\
$\mathrm{~T}_{\text {max }}-\mathrm{F} 60$ & 0.34 & 0.58 & 1.80 & 12.50 & 0.0003 \\
$\mathrm{~T}_{\text {sum }}-\mathrm{E} 120$ & -0.05 & 0.03 & -0.35 & 5.80 & 0.011 \\
$\mathrm{~T}_{\text {sum }}-\mathrm{F} 30$ & 0.04 & -0.19 & 0.50 & 5.60 & 0.012 \\
$\mathrm{~T}_{\text {sum }}-\mathrm{F} 120$ & -0.06 & 0.02 & -0.25 & 4.30 & 0.029 \\
$\mathrm{~T}_{\text {max }}-\mathrm{E} 60$ & 0.21 & -0.37 & -0.40 & 1.70 & 0.209 \\
$\mathrm{~T}_{\text {max }}-\mathrm{E} 30$ & -0.15 & 0.39 & -0.28 & 1.00 & 0.383 \\
Constant & -8.21 & -12.80 & -19.92 & & \\
\hline
\end{tabular}

The variables listed were selected from the 12 original variables in the study by a stepwise process that calculated conditional $F$ ratios and entered one variable at a time starting with the highest $F$ and excluding variables with $F<1$. $F$ ratios and probabilities for all variables selected to compose the discriminant linear function and calculated after the last step are listed. The coefficients of each variable are listed for the three groups studied. When every coefficient was multiplied by the variable value of a given experiment, and all the products and the constant added, a discriminant score, used in classification, was obtained. These scores were transformed to predicted probabilities following the procedure described by James ${ }^{14}$ and are graphically displayed in Figure 5 test. Muscle mass differences would be greatest between the normal and flaccid groups. We, however, find no significant differences in $T_{\max }$ FLX between these groups. In the pendulum test, an electrogoniometer measures the angle of the leg as it drops from full extension $\left(0^{\circ}\right.$ in the convention used in this study) to a resting angle allowing the leg to swing freely. In normal subjects, the leg will swing six or seven times, and in flaccid subjects up to 12 times. ${ }^{8}$ In spastic subjects, however, the strong stretch reflex inhibits the number of oscillations of the leg to only a couple. ${ }^{8,12}$ Further, the oscillations of the spastic group are smaller and dampen quickly, consequently the relaxation index is smaller in the spastic group. The relaxation index is defined as the ratio between the magnitude of the first drop and the magnitude of the difference between initial and final angles. ${ }^{7}$

In the isokinetic dynamometric technique used in this study, the knee is rapidly flexed (moving from $25-75^{\circ}$ - flexing phase of Figure 2) upon transitioning from an extending direction $\left(75-25^{\circ}\right.$ - extending phase of Figure 2). Due to the seated position of the subjects in our configuration (hip position at $90^{\circ}$ ), the hamstrings were maximally stretched at the end of
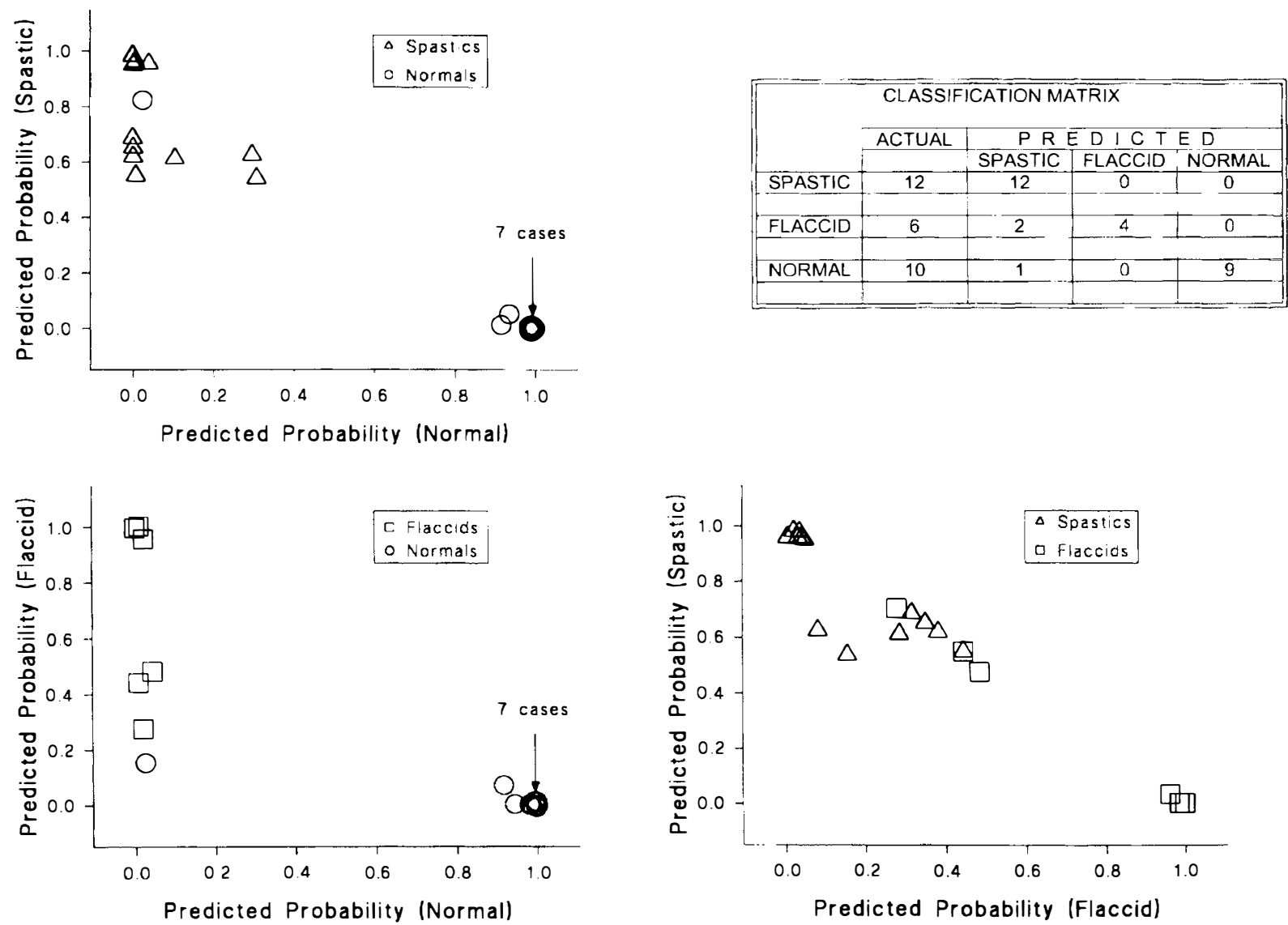

Figure 5 Classification matrix table (right top quadrant) and the three predicted probability graphs for the spastic $v s$. normal groups (left top quadrant), flaccid $v s$. normal groups (left bottom quadrant), and the spastic vs. flaccid group (right bottom quadrant) 
the extending phase $\left(\right.$ at $\left.25^{\circ}\right)$ transitioning into the flexing phase. Consequently, during the rapid transition occurring at $25^{\circ}$ as the lever stopped extending and began flexing, a strong stretch reflex may have been invoked in the hamstrings of the spastic subjects pulling on the shank causing knee flexion while the dynamometer was moving the lever in the flexing direction. Consequently, a smaller negative peak (leg not pulling away from the lever as much) was observed in the spastic group. In the other groups lacking strong stretch reflexes, the inertia properties of the leg continue to accelerate the leg in the extending direction even though the movement of the lever is flexion; thus creating a large negative peak (leg pulling away from the lever) (denoted as F1 in Figure 2).

Although lower $T_{\max }$ FLX values for the spastic group as compared to the normal group are counter to data from other studies, ${ }^{4,12}$ using isokinetic dynamometers, several rationales are possible. First, the testing position used by Lewis ${ }^{12}$ involved patients lying supine (hip angle of $180^{\circ}$, i.e. full extension); thus lengthening the quadriceps. The knee hung over the edge of the Kin-Com chair and muscle tone was evaluated from $0-90^{\circ}$ of flexion $\left(0^{\circ}\right.$ being full extension of the knee). In our study and in that of Firoozbakhsh et al. ${ }^{4}$ subjects were seated with a hip angle of $90^{\circ}$; lengthening the hamstrings instead. It would be expected that $T_{\max }$ FLX values would be larger in the Lewis study ${ }^{12}$ as flexion would further lengthen the already stretched quadriceps. The reverse would be true for $\mathrm{T}_{\max }$ EXT values in which our study and that of Firoozbakhsh et al. ${ }^{4}$ would have larger values in extension since the hamstrings would be further lengthened from an already stretched position. As expected, our study and that of Firoozbakhsh et al. ${ }^{4}$ had larger $T_{\max }$ EXT values than the Lewis study. ${ }^{12}$

Secondly, the Lewis study ${ }^{12}$ used peaks occurring only during the constant velocity phase of the repetition, while our study and the Firoozbakhsh et al. study ${ }^{4}$ used peaks occurring throughout the repetition with $\mathrm{T}_{\max }$ occurring during the acceleratory phase; thus partially accounting for differences in peak $T_{\max }$ values. $T_{\max }$ values were lower in the Lewis study ${ }^{12}$ than for our study and for the Firoozbakhsh et al. study ${ }^{4}$ except for $\mathrm{T}_{\max }$ FLX where the standard deviation was quite large in the Lewis study. ${ }^{12}$ As a result of using only the constant velocity phase, Lewis was able to remove not only the gravitational properties of the limb, but also the inertial properties of the lever; thus also accounting for lower $\mathrm{T}_{\max }$ values. $^{12}$

Thirdly, the patient populations were different between our study and the Lewis ${ }^{12}$ and Firoozbakhsh et $a l .{ }^{4}$ studies. Our study used only complete SCI patients with the majority (9/12) of whom were paraplegics. In the Lewis study ${ }^{12} 6$ of the ten subjects were incomplete and 8 of the ten were quadriplegics. In the Firoozbakhsh et al. study, ${ }^{4}$ there was a heterogeneous patient population including individuals with SCI, stroke, and anterior horn cell disease. The effect of these heterogeneous diagnoses or combining incomplete and complete SCI is unknown. In the Lewis study, ${ }^{12}$ however, the standard deviation was quite large in $\mathrm{T}_{\max }$ FLX. This may reflect the varying effect of incomplete versus complete SCI patients. In the Firoozbakhsh et al. study, ${ }^{4}$ since the hamstrings were lengthened, any differences between their study and our study due to the heterogeneous patient population may be more likely accented in $\mathrm{T}_{\max }$ FLX. This may explain the large differences in $T_{\max }$ FLX between the Firoozbakhsh et al. study ${ }^{4}$ and our study since $\mathrm{T}_{\max }$ values were similar.

Overall, the isokinetic dynamometric technique has been shown to be useful in quantifying spasticity. Caution, however, must be taken to control for testing position. To facilitate clinical use, this study demonstrated that the use of $120^{\circ} / \mathrm{s}$ is sufficient to demonstrate differences between groups. The discriminant analysis using the entire set of variables, however, provides the ability to classify individual subjects to specific groups. This feature could be useful when dealing with assessment of individual responses to therapeutic interventions aimed at modification of spasticity. Further studies are necessary to determine what effect level, extent and, years post injury, as well as different diagnoses will have on this technique.

\section{Acknowledgements}

The authors thank Randi Woodrow, P.T. for help with data collection and Kari Matsumoto, B.S. for help with data analysis. Our research was supported by Grant B603RA from the Department of Veterans' Affairs, Rehabilitation, Research and Development Service.

\section{References}

1 Lehmann JF. Spasticity: Quantitative measurements as basis for assessing effectiveness of therapeutic intervention. Arch Phys Med Rehabil 1989; 70: 6-15.

2 Ashworth B. Preliminary trial of carisoprodol in multiple sclerosis. Practitioner 1964; 192: $540-542$

3 Benecke R, Conrad B, Meinck HM, Holme J. Electromyographic analysis of bicycling on an ergometer for evaluation of spasticity of lower limbs in man. Adv Neurol 1983; 39: 1035-1046.

4 Firoozbakhsh KK, Kunkel CF, Scremin AME, Moneim MS Isokinetic dynamometric technique for assessment of spasticity. $A$ J Phys Med \& Rehab 1993; 72: 379-385.

5 Badj T, Vodovnik L. Pendulum testing of spasticity. J Biomed Eng 1984; 6: $9-16$

6 Bohannon RW, Larkin PA. Cybex II isokinetic dynamometer for documentation of spasticity. Physical Therapy 1985; 65: 46-47.

7 Brown RA, Lawson DA, Leslie GC, Part JN. Observations on the applicability of the Wartenberg pendulum test to healthy, elderly subjects. J Neurol, Neurosurg, Psychiatry 1988; 51: 1171-1177.

8 Price R. Mechanical spasticity evaluation techniques. Critical Reviews in Physical and Rehabilitation Medicine 1990; 2: 65-73.

9 Douglas AJ, Walsh, EG, Wright EW, Edmond P. Muscle tone around the human knee in paraplegia. Quarterly Journal of Experimental Physiology 1989; 74: 897-905. 
10 Walsh EG, Harris P. The use of torque generators to study clonus and disturbances of muscle tone. Paraplegia 1972; 9: 228.

11 Knutsson E. Muscle restraint in speed controlled voluntary and passive movements. In: Kyoto Symposia (EEG Suppl No. 36) Buser PA, Cobb WA, Okuma T, eds. Elsevier Biomedical Press, Amsterdam. 1982; pp 161-168.

12 Lewis CA. A passive joint motion technique for quantification of spasticity. Unpublished Master's thesis. Wright State University, Ohio, 1993.
13 Stefanovska A, Rebersek S, Bajd T, Vodovnik L. Effects of electrical stimulation on spasticity. Critical Reviews in Physical \& Rehabilitation Medicine 1991; 3: 59-99.

14 James M Classification Algorithms. John Wiley \& Sons, New York, 1985 